\title{
System architecture and deployment scenarios for SESAME: Small cEllS coordinAtion for Multi-tenancy and Edge services
}

Ioannis Giannoulakis, George Xylouris, Emmanouil Kafetzakis, Anastasios Kourtis, Jose Oscar Fajardo, Pouria Sayyad Khodashenas, Antonino Albanese, Haralambos Mouratidis, Vassilios Vassilakis

This document is the "accepted article" version of the paper, which has been accepted by IEEE for publication in the workshop Software Defined 5G Networks (Soft5G 2016), within the 2nd IEEE Conference on Network Softwarization (IEEE NetSoft 2016).

Copyright has been transferred to IEEE on 14th May 2016.

Full citation information:

I. Giannoulakis, G. Xylouris, E. Kafetzakis, A. Kourtis, J.O. Fajardo, P.S. Khodashenas, A. Albanese, H. Mouratidis, V. Vassilakis, "System architecture and aspects of SESAME: Small cEIIS coordinAtion for Multi-tenancy and Edge services", 2nd IEEE Conference on Network Softwarization (IEEE NetSoft 2016), Workshop on Software Defined 5G Networks (Soft5G 2016), Seoul, Korea, June 10, 2016.

Links to the published version:

DOI: $\underline{10.1109 / N E T S O F T .2016 .7502483}$

IEEE Xplore: $\underline{\text { http://ieeexplore.ieee.org/xpls/abs all.jsp?arnumber=7502483 }}$

The research leading to these results has received funding from:

- The European Union's H2020 programme, under the Grant Agreement H2020-ICT671596 Research and Innovation Project SESAME - Small cEIIS coordinAtion for Multitenancy and Edge services 


\title{
System Architecture and Deployment Scenarios for SESAME: Small cEllS coodinAtion for Multi-tenancy and Edge services
}

\author{
Ioannis Giannoulakis*, George Xylouris*, Emmanouil Kafetzakis ${ }^{\dagger}$, Anastasios Kourtis*, \\ Jose Oscar Fajardo $\ddagger$, Pouria Sayyad Khodashenas ${ }^{\S}$, Antonino Albanese ${ }^{\Uparrow}$, \\ Haralambos Mouratidis, "Vassilios Vassilakis, " \\ *National Centre for Scientific Research "Demokritos", Greece.\{giannoul, xilouris, kourtis\}@iit.demokritos.gr \\ †ORION Innovations, P.C., Greece $\{$ mkafetz $\} @$ orioninnovations.gr \\ ¥University of the Basque Country, Spain, \{joseoscar.fajardo\}@ehu.eus \\ $\S$ Fundacio i2CAT, Spain,\{pouria.khodashenas\}@i2cat.net \\ IITALTEL S.p.A, Smart Networks \& Products, Italy,\{antonino.albanese $\} @$ italtel.com \\ "University of Brighton, U.K., \{H.Mouratidis, V.Vasilakis\}@ brighton.ac.uk
}

\begin{abstract}
The surge of the Internet traffic with exabytes of data flowing over operators' mobile networks has created the need to rethink the paradigms behind the design of the mobile network architecture. The inadequacy of the 4G UMTS Long term Evolution (LTE) and even of its advanced version LTE-A is evident, considering that the traffic will be extremely heterogeneous in the near future and ranging from $4 \mathrm{~K}$ resolution $\mathrm{TV}$ to machine-type communications. To keep up with these changes, academia, industries and $E U$ institutions have now engaged in the quest for new 5G technology. In this paper we present the innovative system design, concepts and visions developed by the 5G PPP H2020 project SESAME (Small cEIIS coordinAtion for Multi-tenancy and Edge services). The innovation of SESAME is manifold: i) combine the key 5G small cells with cloud technology, ii) promote and develop the concept of Small Cellsas-a-Service (SCaaS), iii) bring computing and storage power at the mobile network edge through the development of nonx86 ARM technology enabled micro-servers, and iv) address a large number of scenarios and use cases applying mobile edge computing.
\end{abstract}

\section{INTRODUCTION}

There is an evident boom of mobile data traffic worldwide which is challenging all paradigms adopted so far in the design of the mobile network architecture. The mass market popularity of smartphones and tablets together with the ever increasing development of new apps and high-definition video services is causing profound modification of what only few years ago was a market dominated by voice traffic and text messages. Indeed, the advances of electronics miniaturization, which keep following the prediction of the Moore's law, made possible to embed in a single mobile device unprecedented computing power and storage capacity. At the same time, this exciting moment is witnessing the development of many new applications such as augmented and virtual reality and new electronic gadgets such wearable devices (e.g. Google glasses). Based on these evidences, vendors such as Ericsson at the end of the first quarter of 2014 registered almost 6.8 billion smartphone subscriptions in total worldwide [1].

Recently, this trend of growth has pushed research institutions, mobile operators, network equipment vendors, regulatory bodies and European institutions to begin the new wave of research efforts which shall lead to 5G technology by 2020 . This joint effort is trying to overcome the evident limitations of the current 4G LTE technology and even of its advanced version LTE-A. Indeed they seem obsolete in order to support exabytes of data traffic with heterogeneous requirements from enhanced Mobile Broadband to MachineType communications. To relieve from what looks like a situation of unpreparedness, the European Commission (EC) have launched a three-phase large Research and Innovation Action (RIA) roadway to $5 \mathrm{G}$ [2].

Currently, there is still uncertainty on how $5 \mathrm{G}$ will look like and several attempts are currently doing the endeavor to shed light onto challenges and research trends [3]. Unlike previous generations, 5G will introduce major changes not only at air interface but also from a flexible network management standpoint. In specific, 5G will take advantage of SoftwareDefined Networking (SDN) and Network Function Virtualization (NFV) principles [4], [5], together with high densification in the Radio Access Network (RAN). SDN and NFV are well established concepts in wired networks (e.g. optical) but they are fairly new concepts for radio communications. The common understanding behind $5 \mathrm{G}$ is that it will be made of a large number of Small Cell (SC) devices. SCs are radio transmitters whose complexity range from just antennas and radio circuits (i.e. remote radio head) up to a full functioning evolved Node B (eNodeB) base station. In general, a SC has a radio finger print much shorter than a conventional cellular base station.

SESAME project [6] falls in the scope of these two principles and promotes the adoption of SCs multitenancy, i.e., multiple network operators will be able to use the SESAME 
platform, each one using his own network 'slice'. Moreover, the idea is to endorse the deployment of SCs with some virtualized functions, with each SC connected to a microserver through appropriate fronthaul technology. A microserver is based on a non-x86 architecture using 64-bit ARMv8 technology. Together with the SC, they form the CloudEnabled Small Cell (CESC) and a number of CESCs form the 'CESC cluster' capable to provide access to a geographical area with one or more operators.

A CESC offers computing, storage and radio resources near the users which can be virtualized. Through virtualization, the CESC cluster can be seen as a cloud of resources which can be sliced to enable multi-tenancy. Therefore, the CESC cluster becomes a neutral host for operators which want to share IT and network resources at the edge of the mobile network. The advantages of the SESAME system is to reduce CAPEX and OPEX for mobile operators, enable users with excellent signal quality when in range of a CESC, and reduce service latency and backhaul costs using dynamic placement of content caches (e.g. video) in the micro-servers. In addition, SESAME promotes the creation of a better ecosystem from the standpoint of SC coordination developing Self-X (self-organizing, selfhealing and self-optimizing) techniques, which are inherent features of the CESCs, and better management of the workload across the CESC cluster.

\section{SESAME SCENARIOS}

\section{A. SESAME Scenario 1}

A typical scenario in which the SESAME system can be exploited is shown in Figure 1. The figure depicts a situation of one CESC provider which owns, deploys and maintains the network infrastructure of Small Cells and Light DC (i.e. ensemble of micro-servers) inside the premises where different enterprises are hosted. In this case the CESC provider shall establish Service Level Agreement (SLA) with each customer enterprise to enable enterprise users to access different services, e.g., Internet access, voice communications, video conferencing, and so on. The deployment of micro-servers can lead to achieve close-to-zero latency, with clear benefits for enhanced Quality of Experience (QoE) of media flows. Indeed this can be achieved resorting to content caching at the level of the Light DC, or in other words storing content at different micro servers locations. It is also worth stressing the fact that in a headquarter hosting different enterprises traffic may fluctuate greatly depending on the time of the day and on the nature of specific events held, thus requiring a flexible system which can be scaled up and down depending on the situation.

As shown in Figure 1, the enterprise scenario will leverage on SESAME features such as intrinsic support of multitenancy since Small Cells operators can provide network services and connectivity over the network owned by the infrastructure provider. Furthermore, SESAME will optimize service delivery to the enterprise end users adapting the network behavior by means of self-organizing network techniques.

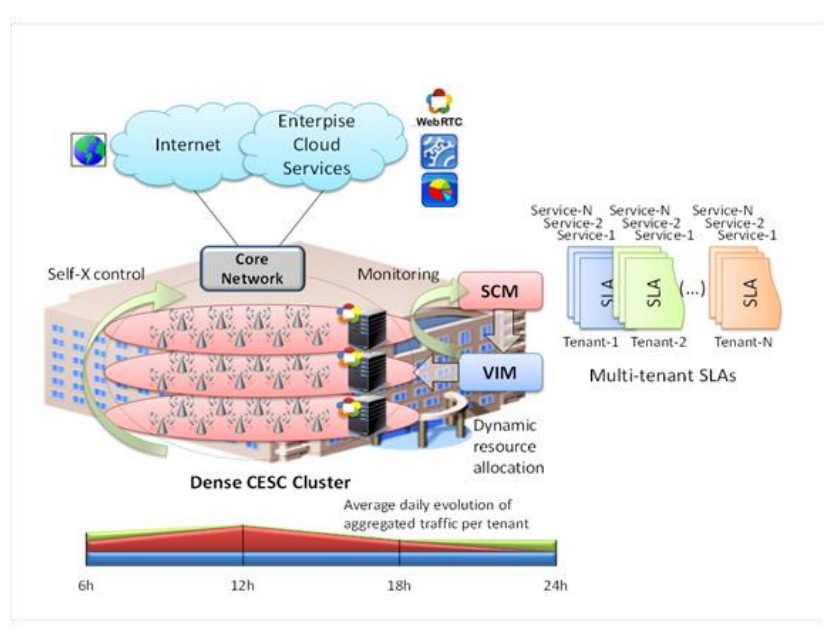

Figure 1. SESAME system for multi-tenancy in enterprise services.

\section{B. SESAME Scenario 2}

In this scenario, a CESC provider manages three distributed CESC clusters deployed in geographically adjacent areas and supports a single mobile network provider that is offering services to his end users through the CESC infrastructures. The relationship between the entities is regulated by different SLAs which are established between the CESC operator and the service provider and between the service provider and its end-users. The main actors and interactions of this scenario are depicted in Figure 2.

To demonstrate different SESAME capabilities in this setup, the mobility of a reference end user is taken into account together with his requirements for service continuity and quality as he moves across the different CESC clusters. The type of traffic generated by the user is assumed to be high-definition real-time content that requires low-latency data access times.

The application of the Mobile Edge Computing (MEC) paradigm, implemented in the SESAME architecture through hardware and software components running at the edge of the network and in the proximity of the moving user, allows for an efficient monitoring of the user location and its related radio conditions, with real-time reporting and the actuation of coordination actions with close to zero latency.

The enhanced handling of the end-user mobility is thus a consequence of the Self-x features of the CESC clusters, which take advantage of edge monitoring capabilities to seamlessly manage the handover process across neighboring cells. As for decision making, limiting the processes within the boundaries of a CESC (or CESCs cluster), allows for the fast application of policies aimed at increasing the overall quality perceived by the user. In order to take into account more stringent requirements (for example across all users), some decision making processes resulting in the reconfiguration of services for the fulfillment of the SLAs can also be completed at the level of the CESCM, which has a wider view of the status of the resources across the CESC clusters.

With respect to user traffic, the scenario highlights how 


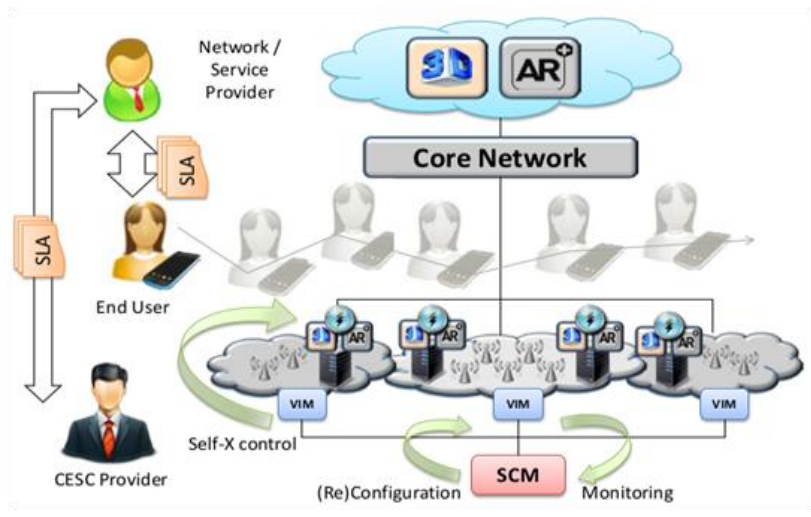

Figure 2. SESAME system for enhanced service experience on the move.

low-latency edge caches can be deployed across the CESC installations to allow the end user an uninterrupted access to content. Pre-provisioning cached data while anticipating the user handover (monitored through signal inspection) is an effective way to offload the user equipments from the task of storing data locally.

\section{SESAME Scenario 3}

A relevant scenario addressed by the SESAME system is presented in Figure 3 where the sudden concentration of people at a specific location creates an unexpected hot spot zone in which a variety of different traffic types require proper management. The sudden gathering of crowds can be due to unexpected live events or emergency situations. This scenario is relevant to leverage on the CESC cluster resources, which is essentially the collection of a number of CESCs (i.e. Small Cells with their micro-servers). In addition, the scenario allows showing that multi-tenancy can be considered a built-in function of the system. In the first place indeed, the CEC infrastructure deployed by an infrastructure provider shall support different mobile operators in serving their customers. In such a situation CESC cluster resources have to be provisioned to each tenant operator, and to handle efficiently the unexpectedly intense traffic generated by the users, selforganizing network techniques are required. At the beginning, the CESCM shall interface with an operator's OSS/BSS to retrieve tenant configuration parameters; afterward, communications and QoS are supported at the CESC level.

It is interesting to notice that this scenario is built on the edge computing capability of the system since computationally intensive tasks can be offloaded from the mobile terminals to the micro-servers, while at the same time optimizing the use of backhaul resources. The two main traffic types which need to be supported and optimized by the CESC cluster are live video streaming (e.g. users film and posting video contents in social media) and real-time group communications (e.g. small community of users exchanging files or videos). Deep packet inspection, video transcoding and data analytics can be enabled in the CESC cluster whereby hardware accelerators in order to optimize the management of the traffic from/to the CESCs.

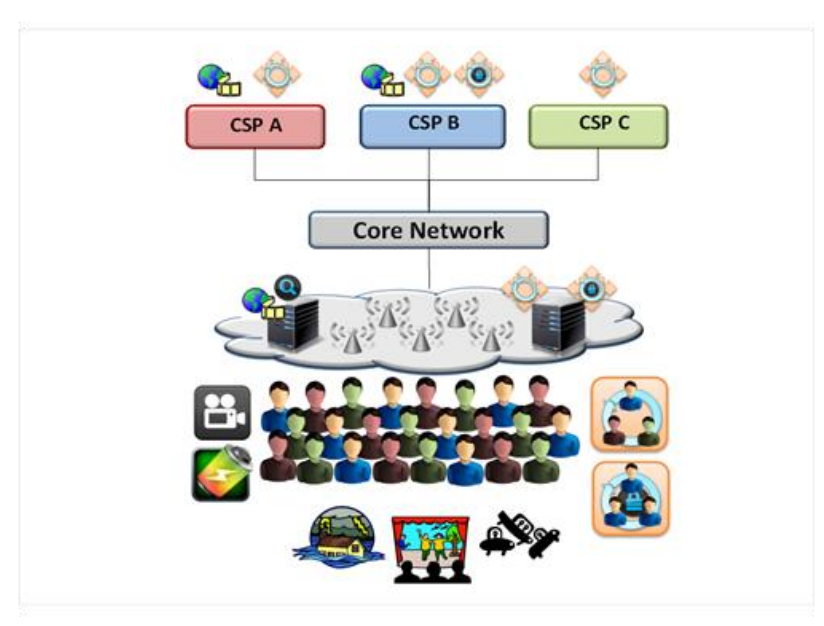

Figure 3. SESAME in flash croud events.

\section{SESAME System ARChitecture}

The key innovations proposed in the SESAME architecture focus on the novel concepts of virtualising Small Cell networks by leveraging the paradigms of a multi-operator (multi-tenancy) enabling framework and an edge-based, virtualised execution environment, see, e.g. Figure 4. Furthermore, SESAME opens the door to 5G system design solutions by investigating, e.g., which core network functions to be virtualized at the edge of the network.

\section{A. CESC}

The CESC includes a multi-tenancy platform able to provide the radio access to support the required wireless capacity in a certain area. In addition, cloud-based computation resources are provided through a virtualised execution platform.. This execution platform is used to support the required Virtualized Network Functions (VNFs) that implement the different features/capabilities of the SCs (and eventually of the core network) and the cognitive/self-x management operations, as well as the computing support for the mobile edge applications of the end-users. An important category of attacks could potentially target this system. Thus, as security will be a fundamental enabling factor of future $5 \mathrm{G}$ networks, we are concerned with identifying and mitigating security threats and vulnerabilities against a broad range of targets at the intersection of NFV, and MEC.

1) Small Cell: Small Cell: the SESAME architecture foresees the split of the small cell physical and virtual network functions, respectively Physical Network Function (PNF) and VNF, based on the Multi-Operator Core Network (MOCN) requirements and associated Radio Resource Manager (RRM) and Operations and Management (OAM) features which need to be supported; Also in scope is to identify, model and analyse security issues from the early stages of system design and software development, as well as to model and analyse threats and vulnerabilities in existing software and protocols that will be used in the SESAME system. 
2) Light Data Centre: Light DC: The SESAME project proposes a micro scale virtualized execution infrastructure in the form of a Light Data Centre (Light DC) to enhance the virtualization capabilities of the Small Cell deployment providing high processing power at the network edge. The Light DC concept provides a high manageable architecture optimized to reduce power consumption, cabling, space and cost. To achieve these requirements it relies on an infrastructure that aggregates and enables sharing of computing, networking and storage resources available in each micro server belonging to the cluster.

The architecture of the micro server is based on a very efficient System on Chip (SoC) that includes multi-core ARMv8 64 bit CPU and HW accelerators dedicated to networking and packet processing. In case of additional computational requirements (e.g. audio and video transcoding, security features, crypto engines), the micro server is able to host standard PCI Express (PCIe) cards equipped with different types of HW accelerators (FPGAs, DSPs, GPUs). In case of high capacity storage requirements (e.g. for video caching) one or more micro servers can host a Disk Controller with the related disks. This feature adds the necessary flexibility for the HW platform to be used with maximum efficiency in various scenarios.

The Light DC infrastructure provides also the backhaul and fronthaul resources for guaranteeing the requirements for connectivity in case of multi-operator (multi-tenancy) scenarios. The hypervisor computing virtualization extensions enables access of virtual machines to the HW accelerators for providing an execution platform that can support the deployment of VNFs. Different types of VNFs can be deployed through the Virtual Infrastructure manager (VIM), for carrying out the virtualization of the Small Cell, for running the cognitive/self-x management operations and for supporting computing needs for the mobile edge applications of the endusers. The combination of the proposed architecture allows to achieve an adequate level of flexibility and scalability in the edge cloud infrastructure.

\section{B. CESC Manager}

In general, CESC Manager (CESCM) is a component with an overall knowledge of the virtual and physical resources, responsible for the deployment, monitoring, configuration and orchestration of the Light DC cloud environment and radio access functionalities, over a single/multiple CESC cluster(s) with a minimum cluster size of one CESC. The main challenge to address is to design a uniform platform where the radio access management task (e.g. transmission power control, packet scheduling, handover and cell reselection thresholds, etc.) and Network Function Virtualization (NFV) management responsibilities (e.g. VNF/service instantiation, lifecycle management, policy management etc.) can be handled in an orchestrated way. Figure 4 depicts a detailed view of the proposed SESAME CESCM which consists of the following modules:

NFV Orchestrator (NFVO): NFVO is responsible for resource orchestration, coordination, authorization, release and

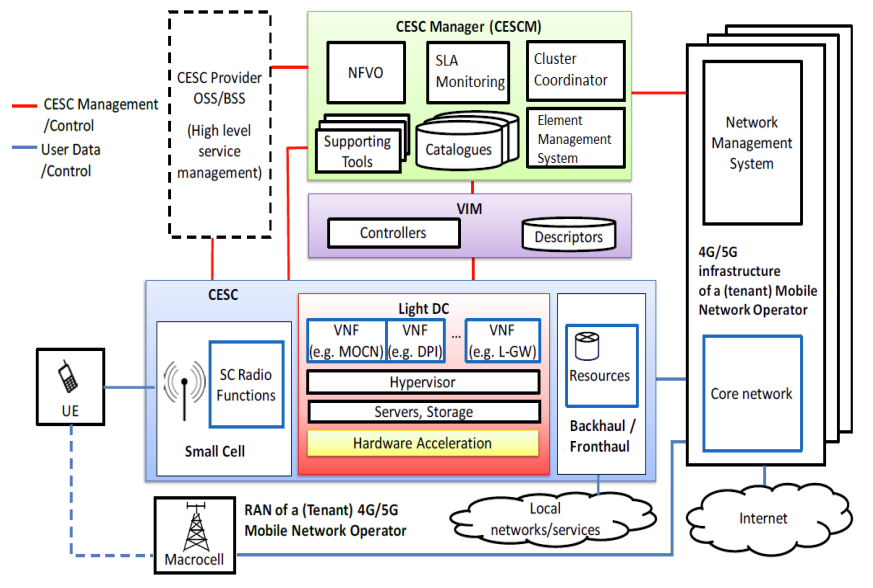

Figure 4. SESAME proposed system architecture.

engage CESC cluster resources via a direct communication with the VIM. The NFVO also undertakes creation, termination, monitoring and scaling of end to end service chains through coordination with respective VNFM(s). Under the scope of SESAME, two type of service chains are identified: i) support service chains composed by only service VNFs without a need for data transmission to the backhaul network, e.g. an edge computation service and ii) network service chains composed by service and SC VNFs supporting data transmission to the backhaul network, e.g. S1-U tunneling between operators EPC and CESCs trough per operator SC VNFs.

SLA Monitoring: It is a real-time monitoring element that allow the enforcement of the agreed SLAs between the different entities (CESC operators, mobile network providers, endusers). Upon the SLA violation, CESCM can apply policies to mandate the reconfiguration, migration, and scale-out of the existing services to comply with the service agreements while dynamically maximizing the utilization of the resources.

Cluster Coordinator: In principle, since infrastructure slicing is possible in SESAME, i.e. among all mounted CESCs in one point of presence, some may form a cluster (thus, a Light DC and radio resources) while the others form another cluster, CESCM contains this element to indicated and dynamically change (if it is necessary) the boundaries of a cluster.

Element Management System (EMS): The key functionality of such an element is divided into five areas - fault, configuration, accounting, performance and security (FCAPS) of physical network functions (PNF), service and SC VNFs. To improve the overall system performance in an automated fashion, EMS functionalities are enriched with holistic self- $x$ features (where $\mathrm{x}$ stands for configuring, healing, optimizing, etc.). For example, self-optimization of CESC radio parameters.

Local catalogues: These are repositories and descriptors to hold detailed information of hardware resources (i.e. storage, switching and computation), VNF descriptors, network service templates and NFV instances. The stored information is used 
for a wide range of purposes including service graph indication to map an incoming edge service request, identifying the service deployment plan and its interconnection and dependencies with already existing services.

\section{Standardization of 5G System Architecture}

The topics covered by the term 5G, currently one of the foremost vehicle of European research activities, cover a large number of topics ranging from the physical too the application layer, and also architectural and management issues. Within this frame SESAME project focuses to both the management and operation of Small Cell deployments but also deals with the management and orchestration of virtualized resources at the edge of the mobile network (i.e. as realized by CESC namely the Base Station and the integrated micro-server). For SESAME architecture design and functionalities several fora such as Standards Development Organizations (SDOs), Industry Specification Groups (ISG) and Open Source initiative are considered. This section attempts an overview of the activities related to $5 \mathrm{G}$ currently performed under the SESAME prism.

\section{A. STANDARDIZATION OF 5 G SYSTEM ARCHITECTURE}

$I T U$ : Standardization work for future $5 \mathrm{G}$ systems has just started. The International Telecommunications Union (ITU) released in September 2015 the overall vision for future 5G systems, which are denoted as IMT-2020 systems in ITU terminology [7]. Based on the observed technology trends, this document settles the expected capabilities beyond current IMT-2000 systems in terms of eight key performance parameters and three usage scenarios (enhanced mobile broadband, ultra-reliable and low latency communications, and massive machine type communications).

It is expected that the standardization efforts will focus on the main areas of enhanced radio technologies and novel system architectures. The former will support new wireless technologies with increased capacities and centralized operation, while the latter will enable an increased efficiency of resources through network softwarization principles.

3GPP: The Third Generation Partnership Project (3GPP) has launched a series of Study Items related to 5G Mobile Network for Advanced communications to be completed in the scope of Release 14 (expected to finish by May 2016) and that will lead to normative work in the scope of Release 15 (expected to freeze in June to December 2018) [8]. Recently 3 GPP has established a liaison with ETSI ISG NFV in order to avoid duplication of work and solve various issues stemming from the challenges occurring from the use of visualization and COTS equipment for the realization of 3GPP core network functionalities. The current issues currently discussed between the NFV IFA WG and the 3GPP SA5 are related to the Fault Management (FM) and Performance Management (PM) data flows as those are handled by the NFVO or the VNFM.

In the scope of the 3GPP, TR 32.842 [9] analyzes different proposed solutions to coordinate the NFV-MANO and the 3GPP management framework. This report is being used as the basis for the normative work that is being developed in the scope of 3GPP Release 14 for management of mobile networks that include virtualized network functions (MAMOVNF) [10]. However, this family of standards are currently restricted to the virtualization of the core network and the IP Multimedia Subsystem.

$S C F$ : Small Cell Forum (SFC) works to remove barriers, drive standards, ensure interoperability and support the deployment of small cells worldwide [2]. SCF is an independent industry and operator association that promotes the deployment of small cells and fosters the standardization of technical aspects specifically related to the field of small cells. In the scope of virtualization of small cells, the SCF has released a number of good technical documents concerning the possible split between physical and virtualized functions [11] or the possible coordination of the network SCF management plane with the ETSI MANO framework [12]. From SESAME perspective, the results of this fora have high impact in the activities related to the implementation of the visualized Small Cell prototype as well as the level of multi-tenancy that will be achieved.

\section{B. ETSI}

ETSI Network Function Visualization ISG: ETSI NFV phase 2 extends the NFV charter, which is now mainly targeted at technology adoption and addressing areas such as testing/validation, performance/assurance, security, stability, interoperability, reliability, availability and maintainability. Collaboration with external bodies is also a key priority for NFV phase 2. From the perspective of SESAME, the most relevant groups for the design of SESAME architecture appear to be the:

IFA (Interfaces and Architecture): The responsibilities of this working group include the delivery of information models and information flows to support interoperability at reference points and the refinement of the architecture and interfaces leading to the production of detailed specifications. As of July 2015, 14 IFA Work Items were active, including the following ones with direct impact on SESAME.

EVE (Evolution and Ecosystem): According to the terms of reference, this working group is responsible to develop feasibility studies and requirements in relation to a) new NFV use cases and associated technical features, b) new technologies for NFV and c) relationship of NFV with other technologies. It should also maintain an overall view of NFV-related work performed elsewhere (e.g. SDOs, industry groups, open source communities) and develop gap analysis on industry standards in areas relevant to NFV.

SESAME work will be influenced by those WG especially tackling issues related to the VNF Descriptor definition for the Small Cell VNFs, SDN and NFV integration at the CESC cluster level (EVE005 Report on SDN Usage in NFV Architectural Framework), VIM resource management for distributed data-center environment and multi-tenancy. SESAME solution takes all these possible solutions into consideration, in order to design a network management system that allows the proper 
deployment and management of the SESAME cloud-enabled small cells.

ETSI MEC: The idea of a computing platform placed at the edge of the mobile network is not new and is carried on inside ETSI by the Mobile-Edge Computing (MEC) Industry Specification Group (ISG) whose activity started on December 2014. It follows the current trends towards cloud-based architectures operating in an IT environment but with the peculiarity of being located at the edge of the mobile network, within the Radio Access Network and in close proximity to mobile subscribers.

MEC platform wants to take advantage from the existing NFV infrastructure - that provides a virtualization platform to network functions enhancing it with new computing/storage resources and creating a virtualization environment for a wide range of applications running at the mobile network edge.

Distinctive features of the MEC architecture are low latency, proximity, location awareness, high bandwidth, realtime insight into radio network information. This facilitates accelerated delivery of content, services and applications from the edge of mobile networks, closer to end-users. The mobile subscriber's experience can be significantly improved through more efficient network and service operations, enhanced service quality, minimized data transit costs and reduced network congestion. In addition, MEC could play a critical role as a market driver supporting the business case for making $5 \mathrm{G}$ a reality, further improving the mobile customer experience.

One of the main goal of ETSI MEC ISG is the specification of an API to interface a MEC Server to an LTE RAN infrastructure, although certain aspects may be more generally applicable to other types of RAN. In this way mobile network operators would be able to provide a standard interface to third-party partners, offering them access to critical features such as location awareness and network context information.

\section{Open Source Initiatives OPNFV}

OPNFV is a carrier-grade, integrated, open source platform to accelerate the introduction of new NFV products and services. The scope of OPNFVs is focused on building NFV Infrastructure (NFVI) and VIM by integrating components from upstream projects such as OpenDaylight, OpenStack, Ceph Storage, KVM, Open vSwitch, and Linux. The project supports also a number of upstream projects with specific objectives. Among those, SESAME may interact and propose its solutions to the following:

Edge NFV $(e N F V)$ articulates the capabilities and behaviors needed in Edge NFV platforms, and how they interact with NFVI and MANO components of NFV solutions. In SESAME the functionalities will be deployed at the very edge of the infrastructure (i.e. in a CESC).

$A R M B$ and: Its role is to integrate and test all aspects of the Brahmaputra release of OPNFV on ARM-based servers. This is very relevant to SESAME since the NFVI will be based on ARM based micro servers, forming a distributed Data Center (DC).
Service Function Chaining (SFC): It provides the infrastructure to install the upstream ODL SFC (i.e ordered list of a network services) implementation project in an NFV environment. The SFC will require traversal of both VNF and PNFs with specific latency bounds and QoS restrictions.

\section{CONCLUSions}

In order to address the needs and requirements of robust and agile network management, and building upon the pillars of network functions virtualisation, mobile-edge computing and cognitive management, SESAMEs goal is the development and demonstration of an innovative architecture, capable of providing Small Cell coverage to multiple operators as a Service. To that end, SESAME envisages to virtualise and to partition Small Cell capacity, while at the same time it aims to support enhanced edge cloud services by enriching Small Cells with micro servers. In the previous section the envisaged use-case scenarios and the proposed system-level architecture have been presented. Additionally, the possible directions for standardization of this effort have been analyzed at the last section of the paper.

\section{ACKNOWLEDGMENT}

This research received funding from the European Unions H2020 Research and Innovation Action under Grant Agreement No.671596 (SESAME project). The work presented here has been a collaborative effort of the SESAME consortium.

\section{REFERENCES}

[1] "Ericsson Mobility Report: On the Pulse of the Networked Society," Ericsson, White Paper, June 2014.

[2] "5G Public Private Partnership (5G PPP)," https://5g-ppp.eu/.

[3] J. Andrews, S. Buzzi, W. Choi, S. Hanly, A. Lozano, A. Soong, and J. Zhang, "What will $5 \mathrm{~g}$ be?" Selected Areas in Communications, IEEE Journal on, vol. 32, no. 6, pp. 1065-1082, June 2014.

[4] D. Kreutz, F. M. V. Ramos, P. Verissimo, C. E. Rothenberg, S. Azodolmolky, and S. Uhlig, "Software-Defined Networking: A Comprehensive Survey," no. 2.01, pp. 1-61, Oct. 2014.

[5] A. Fischer, J. F. Botero, M. Till Beck, H. de Meer, and X. Hesselbach, "Virtual Network Embedding: A Survey," IEEE Communications Surveys \& Tutorials, vol. 15, no. 4, pp. 1888 - 1906, Feb. 2013.

[6] "H2020 5G Project SESAME," http://www.sesame-h2020-5g-ppp.eu.

[7] I. Vision, "Framework and overall objectives of the future development of imt for 2020 and beyond," ITU, Feb, 2014.

[8] “3GPP Work Plan review at Plenary 70," http://www.3gpp.org/ftp/tsgsa/TSG-SA/TSGS-70/Docs/SP-150843.zip.

[9] "3GPP TR 32.842 v13.0.0, Telecommunication management; Study on network management of virtualized networks," September 2015.

[10] "3GPP TR ts 28.500 v0.2.0, Ttelecommunication management; Management concept, architecture and requirements for mobile networks that include virtualized network functions," November 2015.

[11] "Small Cell Forum, Document 106.05.1.01, Virtualization for Small Cells: Overview," June 2015.

[12] "Small Cell Forum, Document 159.05.1.01, Small Cell Virtualization Functional Splits and Use Cases," June 2015. 